\title{
BMC Oral Health Reviewer Acknowledgment 2015
}

Elaine Zhang

\section{Contributing reviewers}

The editors of BMC Oral Health would like to thank all our reviewers who have contributed to the journal in Volume 15 (2015)

Jenny Abanto
Brazil

Yoshihiro Abiko

Japan

Ahmet Hüseyin Acar

Turkey

Guy Adami

USA

Abiola Adeniyi

Nigeria

Joe Aduse-Opoku

UK

Himanshi Aggarwal

India

Hermann Agis

Austria

Bernardo Agostini

Brazil

Sang-Joon Ahn

USA

Jun Aida

Japan

Merve Akcay

Turkey

Mehmet Akin

Turkey
Gisele Caldas Alexandre
Brazil

Ahmad Aljafari

UK

Paul Allison

Canada

Vanessa Alves

Brazil

Manoharan Andiappan

UK

Flaviana Andrade

Brazil

Filippi Andreas

Switzerland

Oleh Andrukhov

Austria

Yulia Anopa

UK

Toshihiro Ansai

Japan

Raquel Antoniazzi

Brazil

Jose Leopoldo Ferreira Antunes

Brazil

Andreza Maria Aranha

Brazil

\section{Thiago Ardenghi}

Brazil

Rodrigo Arthur

Brazil

Paul Ashley

UK

Javier Ata-Ali

Spain

Sheyla Auad

Brazil

\section{Joanna Baginska}

Poland

Carolina Amalia Barcellos Silva Brazil

Sergio Barros

Brazil

Renata Barroso De Carvalho Brazil

Eraldo Batista

Canada

Alexandre Baumgarten

Brazil

Asli Baysal

Turkey

Sema Becerik

Turkey 
Jonas Becktor

Sweden

Georgios Belibasakis

Switzerland

César Bergoli

Brazil

Gabriela Berti

Brazil

Kristina Bertl

Austria

Bishal Bhandari

UK

Vinodh Bhoopathi

USA

Silvio Diego Bianchi

Italy

Lars Bjørndal

Denmark

Fiona Blinkhorn

Australia

Paolo Boffano

Italy

Clarissa Bonifacio

Netherlands

Carolina Borges

Brazil

S. Aida Borges-Yanez

Mexico

Michael Bornstein

Switzerland

Ali Borzabadi-Farahani

UK

Nagihan Bostanci

Switzerland

Mariana Braga

Brazil

Carmela Bresolin

Brazil

Romina Brignardello-Petersen

Canada

Jonathan Broadbent

New Zealand

Josef Bruers

Netherlands
Nurcan Buduneli

Turkey

Bruno Bueno-Silva

Brazil

Yvonne Buischi

USA

Alexandre Bulgarelli

Brazil

Caroline Bulsara

Australia

Sandra Bussadori

Brazil

Azeez Butali

USA

John Butcher

UK

Michael Büttner

Belgium

William Buwembo

Uganda

Marilia Buzalaf

Brazil

Maria Grazia Cagetti

Italy

Rodrigo Cançado

Brazil

Peter Carlsson

Sweden

Thiago Carvalho

Switzerland

Luciano Casagrande

Brazil

Luiza Cassiano

Brazil

Paula Midori Castelo

Brazil

Paula Castelo

Brazil

Talita Castro

Brazil

Frank Catalanotto

USA

Ali Cekici

Turkey
Roger Keller Celeste

Brazil

Benjamin Chaffee

USA

Po-Chun Chang

Taiwan

Ava Chow

Canada

Bruno Chrcanovic

Sweden

Bradley Christian

Australia

Chun-Hung Chu

Hong Kong

Sasanka Chukkapalli

USA

Renata Cimoes

Brazil

Ayse Basak Cinar

Denmark

Georg Conrads

Germany

David Conway

UK

Marcos Britto Correa

Brazil

Marcos Corrêa

Brazil

Patrícia Corrêa-Faria

Brazil

Luciane Costa

Brazil

Yuri Costa

Brazil

Luis Cota

Brazil

Leonard Crocombe

Australia

David Crowe

USA

Carla Cugini

USA

Carolina Ortigosa Cunha Brazil 
Cristiane Da Mata

Ireland

Dorothea Dagassan

Switzerland

Anna Damanaki

Cees De Baat

Netherlands

Thiago De Marchi

Brazil

Patricia De Palma

Sweden

Harsha De Silva

New Zealand

Teun J. De Vries

Netherlands

Alberto Delbem
Brazil

Dong Deng

Netherlands

James Deschner

Germany

Matthias Dettmer

Switzerland

Ana Paula Dias Ribeiro

Brazil

Christina Diogo Löfgren

Sweden

Rafael Ditterich

Brazil

Kimon Divaris

USA

Ezgi Doğanay

UK

Ewa Dolinska

Poland

Sophie Doméjean

France

Mojtaba Dorri

Iran, Islamic Republic Of

Jennifer Doss

Malaysia

Poliana Duarte

Brazil
Joyce Duarte

Brazil

Denise Duijster

Netherlands

Walter Dukic

Croatia

Alexandrina Lizica Dumitrescu

Norway

Tom Dyer

UK

Kurt Ebeleseder

Austria

Sigrun Eick

Switzerland

Kim Ekstrand

Denmark

Tarek El-Bialy

Canada

Azza El-Housseiny

Egypt

Arjuna Ellepola

Kuwait

Bruno Emmanuelli

Brazil

Stefano Eramo

Italy

Gülfem Ergün

Turkey

Vanessa Euzebio Alves

Brazil

Wendell Evans

Australia

Megan Falsetta

USA

Ali Farahani

UK

Gisele Faria

Brazil

Nasim Fazel

USA

Georg Feichtinger

UK

Robert Fell

Australia
Giovana Fernandes

Brazil

Karin Fernandes

Brazil

Maurizio Ferrante

Italy

Patricia Ferrari

Brazil

Efigênia Ferreira

Brazil

Carlos Marcelo Figueredo

Brazil

John Firriolo

USA

Edgar-Arturo Flores-Larios

Mexico

Morenike Folayan

Nigeria

Lyndie Foster Page

New Zealand

Ashraf Fouad

USA

Deng Fu

China

Piotr Fudalej

Switzerland

Tsuyoshi Fujita

Japan

Peter Gaengler

Germany

Ana Gamboa

UK

Jinlong Gao

Australia

Iad Gharib

UK

Barry Gibson

UK

Fiona Gilchrist

UK

Thais Gimenez

Brazil

Nikolaos Gkantidis

Switzerland 
Werner Goetz

Germany

Ali Golkari

Iran, Islamic Republic Of

Viviane Elisângela Gomes

Brazil

Lucio Gonçalves

Brazil

Raúl González-García

Spain

Morris Gordon

UK

Melissa Grant

UK

Ana Flavia Granville-Garcia

Brazil

Reinhard Gruber

Austria

Renata Guedes

Brazil

Pinar Gumus

Turkey

Orachad Gururatana

Thailand

Alex Nogueira Haas

Brazil

Sebastian Hahnel

Germany

Hassan Halawany

Saudi Arabia

Demetrios Halazonetis

Greece

Violet Haraszthy

USA

Melanie Hayes

Australia

Chuanglong He

China

Lisa Heaton

USA

Eva Hedman

Sweden

Rebecca Helmreich

USA
Min-Suk Heo

Korea, South

Bruno S Herera

Brazil

Daniela Hesse

Brazil

Nora Hiivala

Finland

Bruce Hirsch

USA

Ha Hoang

Australia

Kristin Hoeft

USA

Marinella Holzhausen

Brazil

Kiyonobu Honma

USA

Stephen Hsu

Singapore

Hsiao-Ling Huang

Taiwan

Michael Hülsmann

Germany

Haizal Hussaini

New Zealand

Koji Inagaki

Japan

Nicola Innes

UK

Andrei Ionescu

Italy

Kazuyuki Ishihara

Japan

Atsonori Isozaki

Japan

Kanade Ito

Japan

Masanori Iwasaki

Japan

Yuichi Izumi

Japan

Reinhilde Jacobs

Belgium
Joanna Janiszewska-Olszowska

Poland

Claude Jaquiery

Switzerland

Juliana Jardim

Brazil

Fawad Javed

USA

Newell Johnson

Australia

Birgitta Jönsson

Norway

Beuy Joob

Thailand

Horng-Heng Juang

Taiwan

Roger Junges

Norway

Luis Junquera

Spain

Jessica Kajfasz

USA

Thomas George Kallarakkal

Malaysia

Atsushi Kameyama

Japan

Ines Kapferer

Austria

Ertugrul Karatas

Turkey

Matthias Karl

Germany

Konstantinos Katsoulis

Switzerland

Mehmet Emin Kaval

Turkey

Katerina Kavvadia

Greece

Hakki Oguz Kazancioglu

Turkey

Moritz Kebschull

Germany

Ali Keles

Turkey 
Muhammad Khalil Khan

Saudi Arabia

Kyeong-Seop Kim

Korea, South

Shiho Kino

Japan

Shiho Kino

UK

Atsuhiro Kinoshita

Japan

Mitsuo Kishi

Japan

Yuichi Kitasako

Japan

Marlise Klein

Brazil

Marlise Klein

USA

Cristiane Koga-Ito

Brazil

Klaus König

Netherlands

Dorota Kopycka-Kedzierawski

USA

Sudaduang Krisdapong

Thailand

Jeroen Kroon

Australia

Sebastian Kühl

Switzerland

Katherine Kula

USA

Wayne Kye

USA

Elena Labajo González

Spain

Giuseppina Laganà

Italy

Ratilal Lalloo

Australia

Jerónimo Lazos

Argentina

Soraya Leal

Brazil
Song Lee

Canada

Gillian Lee

Hong Kong

André Leite

Brazil

Claudio Leles

Brazil

Edina Lempel

Hungary

Tathiane Lenzi

Brazil

Roos Leroy

Belgium

Charlotte Lewis

USA

Ming-Yu Li

China

Lingling Li

USA

Sungwoo Lim

USA

Ramille Lima

Brazil

Huan-Cai Lin

China

Tülay Lindberg

Sweden

Ulrika Lindmark

Sweden

Giovanni Lodi

Italy

Carl Lombard

South Africa

Tommaso Lombardi

Switzerland

Peter Loomer

USA

Bruno Loos

Netherlands

Rodrigo Lopez

Denmark

Lucianne Maia

Brazil
Arash Mansourian

Iran, Islamic Republic Of

Medeiros Mara

Mexico

Rodrigo Mariño

Australia

Merete Markvart

Denmark

Zoe Marshman

UK

F.S. Martelli

Italy

Conchita Martin

Spain

Maria Carolina Martins Mussi

Brazil

Stephen Mason

UK

Manu Mathur

India

Yusuke Matsuyama

Japan

Juliana Mattos-Silveira

Brazil

Joanna May

UK

Mara Medeiros

Mexico

Noshir Mehta

USA

May Mei

Hong Kong

Peter Meisel

Germany

Mahtab Memarpour

Iran, Islamic Republic Of

Michel Messora

Brazil

Theodorus (Dirk) Mettes

Netherlands

Hendrik Meyer-Lückel

Germany

Fabio Mialhe

Brazil 


\begin{tabular}{|c|c|c|}
\hline $\begin{array}{l}\text { Margaret Miller } \\
\text { Australia }\end{array}$ & $\begin{array}{l}\text { Rahul Nair } \\
\text { Singapore }\end{array}$ & $\begin{array}{l}\text { Anna-Lena Ostberg } \\
\text { Sweden }\end{array}$ \\
\hline Steve Miller & Orawan Nammontri & Kazuhisa Ouhara \\
\hline USA & Thailand & Japan \\
\hline Kyung-San Min & A Maya Nandkumar & Elizabeth Oziegbe \\
\hline Korea, South & India & Nigeria \\
\hline Richard Miron & Keiko Naruse & Damla Özsu \\
\hline Canada & Japan & Turkey \\
\hline Eduardo Moffa & Gustavo Nascimento & V. Özgen Öztürk \\
\hline Brazil & Brazil & Turkey \\
\hline Osman Mohamed Osama Osman & Declan Naughton & Keerthilatha Pai \\
\hline Switzerland & UK & India \\
\hline Anelise Montagner & Robert Nedelcu & Nikolaos Pandis \\
\hline Brazil & Sweden & Switzerland \\
\hline Deborah Moore & Tim Newton & Gianpaolo Papaccio \\
\hline UK & UK & Italy \\
\hline Rafael Moraes & Dominique Niesten & Moschos Papadopoulos \\
\hline Brazil & Netherlands & Greece \\
\hline John Moran & Peter Nijkamp & George Papantonopoulos \\
\hline UK & Netherlands & Greece \\
\hline Carlos Moreira & Yoshikaki Nomura & Masoud Parirokh \\
\hline Brazil & Japan & Iran \\
\hline Annie Morgan & Ryota Nomura & Renato Parsekian Martins \\
\hline UK & Japan & Brazil \\
\hline Susan Morison & Hiraishi Noriko & Shankargouda G. Patil \\
\hline UK & Japan & India \\
\hline Toshiya Morozumi & Shimizu Noriyoshi & Antonio Carlos Pereira \\
\hline Japan & Japan & Brazil \\
\hline Gordon Morris & Ana Nunes & Luciano Pereira \\
\hline UK & Brazil & Brazil \\
\hline Alireza Moshaverinia & Enihomo Obadan-Udoh & Karen Peres \\
\hline USA & USA & Australia \\
\hline Jan Mulder & Yorimasa Ogata & Marco Peres \\
\hline Netherlands & Japan & Australia \\
\hline Heinz Müller & Taiji Ogawa & Juliano Pessan \\
\hline Switzerland & Japan & Brazil \\
\hline Wondemagegn Mulu & Takahiko Oho & Ove Peters \\
\hline Ethiopia & Japan & USA \\
\hline Gonca Mumcu & Guilherme Oliveira & Elizabeth Philipone \\
\hline Turkey & Brazil & USA \\
\hline Brian Muzyka & Denise Oliveira & Lucas Pinheiro \\
\hline USA & Brazil & Brazil \\
\hline Rahul Naidu & Lucy O'Malley & Ericka Pinheiro \\
\hline Trinidad And Tobago & UK & Brazil \\
\hline
\end{tabular}


Chaiana Piovesan

Brazil

Fabiane Piva

Brazil

Antonella Polimeni

Italy

Deborah Polk

USA

Andre Priede

Australia

Laura Primo

Brazil

Sumant Puri

USA

Parth Purwar

India

Gert Jan Putten

Netherlands

Maryam Rabiei

Iran

Daniela Raggio

Brazil

Smrithi Rajendiran

USA

Karthikeyan Ramalingam

India

Joana Ramos-Jorge

Brazil

Cameron Randall

USA

Sarbin Ranjitkar

Australia

Amornrat Ratanasiri

Thailand

Nilantha Ratnayake

Sri Lanka

Daniel Reissmann

Germany

Torsten W. Remmerbach

Germany

Daniela Rios

Brazil

Agneta Robertson

Sweden
Peter Robinson

UK

Rachel Rocha

Brazil

Laura Roesch

Mexico

Matthias Roggendorf

Germany

Ellen Rogo

USA

Marina Roscoe

Brazil

Martin Rosentritt

Germany

Gabriele Rossini

Italy

Rodnei Dennis Rossoni

USA

Patrick Rouxel

UK

R Gary Rozier

USA

Ingrid Rozylo-Kalinowska

Poland

Jianping Ruan

China

Thiago Saads Carvalho

Switzerland

Omer Sagsoz

Turkey

Philipp Sahrmann

Switzerland

Meenu Saini

USA

Atsushi Saito

Japan

Christian Salazar

USA

Katayoun Salem

Iran

Humam Saltaji

Canada

Vidya Sankar

USA
Mauro Pedrine Santamaria

Brazil

Oseas Santos Junior

Brazil

Terukazu Sanui

Japan

Yukihiro Sato

Japan

Anurag Satpathy

India

Edgar Schäfer

Germany

Benoit Schaller

Switzerland

Rob Mh Schaub

Netherlands

Andrea Maria Schmidt-Westhausen

Germany

Anja Schmitt

Switzerland

Dirk Schulze

Germany

Joel Schwartz

USA

Uwe Schwarze

Austria

Don Schwass

New Zealand

Helmut Schweikl

Germany

Falk Schwendicke

Germany

David Scott

USA

Hidenobu Senpuku

Japan

Benedict Seo

New Zealand

Junia Serra-Negra

Brazil

Salvatore Settineri

Italy

Rabia Gonul Sezer

Turkey 
Siddharth Shanbhag

India

Koki Shioya

Japan

Bernd W. Sigusch

Germany

Emmanuel Silva

Brazil

Lucas Hian Silva

Brazil

Cleverson Silva

Brazil

Chelsia Sim

Singapore

Alyne Simoes

Brazil

Ankur Singh

Australia

Ripudaman Singh

India

Shenuka Singh

South Africa

Carla Sipert

Brazil

Gustavo Sivieri-Araujo

Brazil

Anna Skalniak

Poland

Marit S Skeie

Norway

Anna Skurska

Poland

Linda Slack-Smith

Australia

Adriana Soares

Brazil

Francesca Soldani

UK

Mikael Sonesson

Sweden

Gianrico Spagnuolo

Italy

Heiko Spallek

USA
Alexandra Stähli

Switzerland

Joao Paulo Steffens

Brazil

Patricia-Anca Steinmassl

Austria

Emelie Stenberg

Sweden

Simon Stone

UK

Stefan-Ioan Stratul

Romania

Juliana Stuginski-Barbosa

Brazil

Rüstem Kemal Sübay

Turkey

Karin Sunnegårdh-Grönberg

Sweden

Nao Suzuki

Japan

Koichi Tabeta

Japan

Hiroyuki Tada

Japan

Santosh Kumar Tadakamadla

India

Anwar Tappuni

UK

Tamara Tedesco

Brazil

Tellervo Tervonen

Finland

Thomas Thurnheer

Switzerland

Tamanna Tiwari

USA

Mimmi Tolvanen

Finland

Fernanda Tomazoni

Brazil

Utsana Tonmukayakul

Australia

Huseyin Topcuoglu

Turkey
Hüseyin Sinan Topçuoglu

Turkey

Lidia Tordiglione

Italy

Markus Troeltzsch

Germany

Simone Tuchtenhagen

Brazil

Anne Bjørg Tveit

Norway

Sergio Uribe

Chile

Vinay V Kumar

India

Diego Valentim

Brazil

Monique Van Der Veen

Netherlands

Denise Van Diermen

Netherlands

Fabiana Vargas-Ferreira

Brazil

Carlalberta Verna

Switzerland

Christopher Vernazza

UK

Lance Vernon

USA

Mario Vettore

UK

Stella Vieira

Brazil

Alexandre Rezende Vieira USA

Rui Miguel Pinheiro Vitorino Portugal

Rodrigo Vivan

Brazil

Ana Vukovic

Serbia

John Walters

USA

Zilu Wang

China 
Shuai Wang

Hong Kong

Qian Wang

USA

Saman Warnakulasuriya

UK

Michael Wefelmeier

Germany

Florian J. Wegehaupt

Switzerland

Lam Wendy

Hong Kong

Annette Wiegand

Germany

Tove Irene Wigen

Norway

Leslie Will

USA

Brita Willershausen

Germany

Peter Windisch

Hungary

Sarah Wong

Hong Kong

Raymond Wong

Singapore

Kyung Mi Woo

Korea, South
Devina Worsley

UK

Jibieke Wulaerhan

China

Alidianne Xavier

Brazil

Masaru Yamaguchi

Japan

Tatsuo Yamamoto

Japan

Duygu Yaman

Turkey

Yan Yang

China

Ghaeth Yassen

USA

Ibrahim Yaylali

Turkey

Ping Ye

Australia

Veerasamy Yengopal

South Africa

Anthony Yeo

Australia

Akihiro Yoshida

Japan

Rodrigue Yossa Nouaga

Canada
Rosnah Binti Zain

Malaysia

Carlos Zaror

Chile

Piero Antonio Zecca

Italy

Florian Zeman

Germany

Lin Zeng

USA

Wenjian Zhang

USA

Lai-Ping Zhong

China

Zhi Zhou

China

Xiaofei Zhu

China

Thomas Ziebart

Germany

Stefan Zimmer

Germany

Brigitte Zimmerli

Switzerland

Argyro Zougli

Germany 